\title{
La Instrucción en Procesos Mecánicos y Sustantivos de la Escritura a través de las Tecnologías de la Información y la Comunicación (TIC): Una Revisión Empírica
}

\author{
María Victoria González Laguna* y Raquel Fidalgo Redondo \\ Universidad de León
}

\author{
PALABRAS CLAVE \\ Nuevas tecnologías \\ Escritura \\ Herramientas digitales \\ Composición escrita \\ Expresión escrita
}

\begin{abstract}
RESUMEN
La escritura es una habilidad esencial que permite al ser humano comunicarse y aprender, por lo que su buen desarrollo favorece el éxito académico y laboral. Actualmente, la inmersión de las nuevas tecnologías en la sociedad ha llevado a la reformulación de las prácticas de instrucción en escritura, siendo necesario el conocimiento y el empleo de un gran número de recursos tecnológicos para poder redactar y comunicarse de manera eficaz. Como consecuencia, durante los últimos años se han creado múltiples recursos digitales para facilitar la adquisición de la competencia escrita. Este estudio tiene como objetivo realizar una revisión de artículos empíricos en los que se utilizan herramientas digitales que han sido diseñadas para mejorar las habilidades de escritura de los estudiantes. Para ello, se ha realizado un análisis de los mismos, obteniendo información sobre las muestras, las variables evaluadas y los resultados obtenidos, así como de los límites y lagunas de conocimiento que sentarán las bases para futuras líneas de investigación. Concluyendo, a nivel educativo, la presente revisión puede ser de utilidad para que el profesorado conozca e incorpore en sus clases distintas herramientas digitales destinadas a la mejora de la competencia escrita.
\end{abstract}

\section{Instruction in Mechanical and Substantive Writing Processes through TIC: An Empirical Review}

New technologies

Writing

Digital tools

Written composition

Written expression

\begin{abstract}
Writing is an essential skill that allows human beings to communicate and learn, and its good development favours academic and work success. Currently, the immersion of new technologies in society has led to the reformulation of writing instruction practices, requiring the knowledge and use of a large number of technological resources in order to be able to write and communicate effectively. As a consequence, many digital resources have been created in recent years to facilitate the acquisition of writing skills. The aim of this study is to review empirical articles in which digital tools designed to improve students' writing skills are used. To this end, an analysis of these articles has been carried out, obtaining information on the samples, the variables evaluated and the results obtained, as well as the limits and gaps in knowledge that will lay the foundations for future lines of research. In conclusion, at an educational level, this review may be useful for teachers to learn about and incorporate different digital tools aimed at improving writing skills in their classes.
\end{abstract}

Universidad de León

Autora de correspondencia: * María Victoria González Laguna; mgonzl@unileon.es

Recibido: 07/05/2021 - Aceptado: 15/09/2021

Revista de Formación del Profesorado e Investigación Educativa

Facultad de Formación del Profesorado y Educación

Universidad de Oviedo

Enero - Diciembre 2021

ISSN: $2340-4728$

(c) (1) () $\odot$ Esta obra está bajo una licencia internacional Creative Commons

Atribución-NoComercial-SinDerivadas 4.0. 


\section{Introducción}

La escritura es una herramienta fundamental en el proceso de enseñanza-aprendizaje del alumnado, ya que no solo permite la adquisición y la elaboración del conocimiento, sino que también da la oportunidad de difundir y comunicar todo aquello que ha sido adquirido durante el proceso aprendizaje (Graham y Perin, 2007; Suárez-Palacio et al., 2018). Por ello, el desarrollo de la competencia escrita se ha convertido en una de las principales preocupaciones para el profesorado al llevar a cabo la alfabetización del alumnado, porque el logro de un buen dominio de las habilidades de escritura favorecerá el éxito académico y, posteriormente, el laboral (González-Álvarez, 2018; Graham \& Perin, 2007; Graham et al., 2017; Kwak, 2017).

A pesar de que algunos autores consideran que la enseñanza de la escritura es una de las principales habilidades cuya enseñanza debe favorecerse en las escuelas desde edades tempranas (Suárez-Palacio et al., 2018), en múltiples estudios se ha puesto de manifiesto que existe un gran número de estudiantes que llegan a la Educación Superior con un mal dominio de sus habilidades de escritura (González-Álvarez, 2018; Kwak, 2017). Asimismo, recogen que una de las posibles causas de ese mal desempeño en escritura puede estar relacionada con una enseñanza inadecuada de la misma en los centros educativos.

En las últimas décadas, se han realizado diversos meta-análisis con el fin de obtener información sobre las prácticas instruccionales de escritura existentes $\mathrm{y}$, más concretamente, sobre su eficacia. En dichos estudios, se ha mostrado que los programas de instrucción estratégica y autorregulada son los más eficaces para mejorar la competencia escrita, tanto del alumnado con dificultades de aprendizaje (Gillespie y Graham, 2014; Graham et al., 2013) como del alumnado sin dificultades (Graham y Perin, 2007; Koster et al., 2015).

Actualmente, también ha cobrado importancia la influencia de las nuevas tecnologías en la práctica instruccional de la escritura, ya que su inmersión en la sociedad ha cambiado la manera de comunicarnos, transformando así la naturaleza de las prácticas de alfabetización. En el contexto escolar la escritura va más allá del lápiz y el papel, requiriendo el conocimiento y la utilización de un gran número de medios tecnológicos que permiten al individuo comunicarse y redactar de una manera eficaz (Andrade y Moreno, 2017; Baker y Lastrapes, 2019; Bolaño-García, 2017). Sin embargo, en muchas ocasiones los equipos docentes carecen de los conocimientos, estrategias y habilidades necesarias para introducir las nuevas tecnologías en los programas de instrucción en escritura (Leu et al., 2013; Wang et al., 2014). Según el estudio realizado por Venegas-Ramos et al. (2020) con una muestra de 69 docentes chilenos en ejercicio, solo 12 de ellos $(17,39 \%)$ afirmaron haber recibido formación formal sobre Tecnologías de la Información y de la Comunicación (TIC), mientras que el resto adquirieron sus conocimientos de manera autodidacta o por mediación de sus compañeros. En este sentido, España es uno de los países con mayor nivel de formación en TIC, ya que el informe TALIS 2018 muestra que un $90 \%$ de los docentes ha recibido formación formal sobre TIC, mientras que en otros países como Inglaterra y Corea se reduce al 50\% (Ministerio de Educación y Formación Profesional, 2019). Asimismo, otro dato llamativo sobre España es que, según el informe TALIS 2018 (Ministerio de Educación y Formación Profesional, 2019), el uso de las TIC por parte de los docentes varía entre las comunidades autónomas, siendo Cantabria, La Rioja y Castilla y León las que menos utilizan las TIC en el aula.

En los últimos años, se ha producido un aumento del desarrollo de herramientas tecnológicas centradas en la mejora de la escritura y, por ende, en la investigación sobre su efectividad (Limpo et al., 2020; Little et al., 2018). Las TIC aplicadas a la instrucción en escritura han mostrado ser eficaces para la mejora de la calidad textual, tal y como se ha reflejado en revisiones centra- das en investigar la instrucción en escritura tanto en estudiantes con dificultades de aprendizaje, como la realizada por Perin y Holschuh (2019), como en población normalizada de Educación Secundaria o Educación Superior, como se demuestra en las revisiones empíricas de Galvin y Greenhow (2019) y Strobl et al. (2019). Sin embargo, no hay datos sobre su eficacia en Educación Infantil y en Educación Primaria. A su vez, en revisiones como la elaborada por Rodríguez et al. (2019), se ha ofrecido un listado de herramientas digitales para la enseñanza de estrategias y procesos de escritura de orden superior, pero todas eran Sistemas de Tutoría Inteligente (STI). Por lo tanto, se dejaban de lado otro tipo de herramientas digitales de utilidad, como las presentadas por Rowland et al. (2020) para mejorar procesos mecánicos de la escritura o los Sistemas de Evaluación de Escritura Automatizada (AWE), que sirven para mejorar los procesos de orden superior en escritura.

\section{Objetivos}

El objetivo del presente estudio es realizar una revisión sistemática de artículos empíricos centrados en la utilización de herramientas digitales, focalizadas tanto en procesos de alto, como de bajo nivel cognitivo de escritura, que han sido diseñadas para mejorar la calidad textual de las composiciones escritas del alumnado. Con su realización se pretende conocer el estado de la cuestión sobre el uso de las TIC en la instrucción en escritura, analizando las herramientas TIC disponibles para instruir en procesos de alto y de bajo nivel cognitivo y su aplicabilidad en función del tipo de muestra.

Desde nuestro conocimiento las revisiones previas que estudian el uso de las TIC para mejorar la escritura se han centrado en las etapas de Educación Secundaria y Educación Superior (Galvin y Greenhow, 2019; Strobl et al., 2019), frente a las etapas iniciales de escolarización. Quizá este hecho, puede reflejarse en que las etapas educativas en las que se realizan menos estudios sobre la influencia de las TIC en la composición escrita son Educación Infantil y Primaria, lo que se plantea como primera hipótesis. Asimismo, revisiones como la de Limpo et al. (2020), en las que se ofrece un listado de herramientas TIC aplicadas a la instrucción escrita, reflejan que la mayor parte de las herramientas existentes se centran en mejorar aspectos como la gramática. Por lo tanto, la segunda hipótesis es que esta misma tendencia se observa a lo largo de toda la escolarización pre y postobligatoria, esperando hallar un mayor número de recursos digitales focalizados en aspectos mecánicos de escritura, frente a los centrados en procesos de orden superior.

\section{Metodología}

\section{Procedimiento de búsqueda y selección documental}

La búsqueda inicial se realizó en lengua inglesa mediante las bases de datos Web of Science (WOS) y Scopus, con el fin de localizar artículos empíricos que abordaran la temática de la mejora de la escritura a través de herramientas TIC o herramientas digitales.

Para localizar dichos artículos, se emplearon diversas sintaxis de búsqueda combinando palabras clave como, por ejemplo: writing instruction AND new technologies, writing intervention AND technology, technology-enhanced learning AND writing o intelligent tutoring system AND writing.

En cada búsqueda, en primer lugar, se seleccionaron todos aquellos documentos que guardaban una relación directa con la temática a tratar. Para ello, se hizo una lectura del título, resumen y objetivo perseguido en los distintos estudios. En segundo lugar, tras descartar los artículos repetidos o duplicados, se aplicaron una serie de criterios de inclusión, con el fin de seleccionar exclusivamente aquellos documentos que se ajustaran a los mismos. Los criterios empleados fueron: $a$ ) texto completo; $b$ ) centrado en 
alumnado de cualquier nivel educativo (Educación Infantil, Educación Primaria, Educación Secundaria y Educación Superior); c) utilizar una herramienta digital para mejorar la composición escrita; d) artículo empírico y e) escrito en inglés o en español. Finalmente, según los criterios establecidos, se seleccionaron un total de 76 artículos empíricos.

El procedimiento seguido para la búsqueda y la selección documental aparece reflejado en la Figura 1 mediante un diagrama de flujo PRISMA.

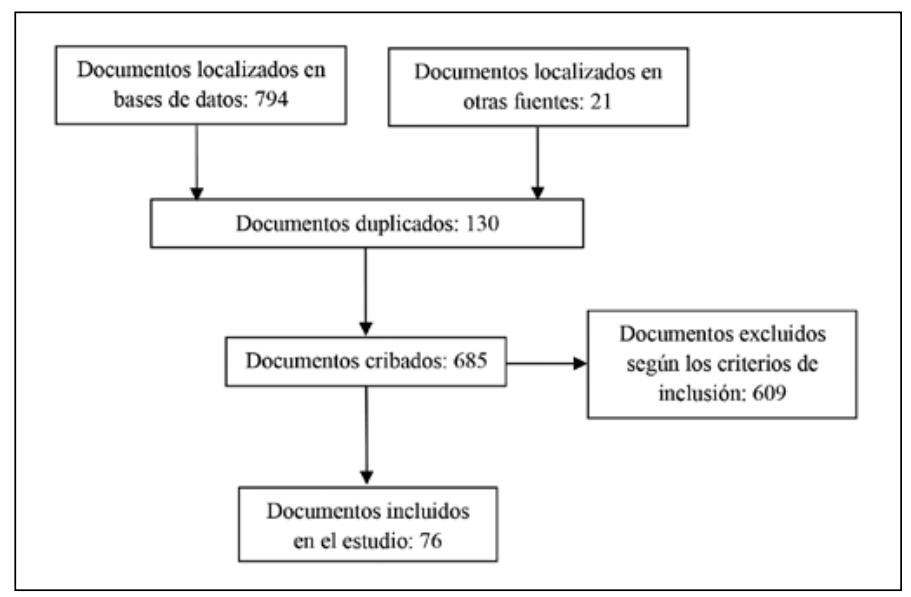

Figura 1. Procedimiento de búsqueda y selección documental

\section{Procedimiento de análisis}

Una vez finalizada la búsqueda y la selección de documentos, se llevó a cabo el análisis de los mismos. En primer lugar, se realizó un vaciado de datos de todos los estudios seleccionados, extrayendo la información relacionada con el objetivo, la muestra, los recursos tecnológicos, las variables evaluadas y los resultados obtenidos.

En segundo lugar, se organizaron los artículos en dos dimensiones: por un lado, aquellos en los que se empleaba un recurso digital para mejorar aspectos mecánicos o de bajo nivel cognitivo de escritura. Por otro lado, los artículos en los que el recurso digital se utilizara para la enseñanza de procesos de orden superior.

Por último, se recogió la información obtenida mediante tablas sintetizadas, en las que aparecen reflejados los aspectos comunes entre los distintos estudios empíricos.

\section{Muestra}

En esta revisión se analizaron 76 estudios empíricos, de los cuales el 61,8 \% fueron realizados entre 2021 y 2016; el 19,7\% entre 2015 y 2010; y el 18,4 \% entre 2009 y 1994; lo que refleja el avance significativo del interés de las TIC y su aplicación al ámbito educativo, concretamente a la escritura, en los últimos años.

A continuación, se exponen los resultados obtenidos tras el análisis de los documentos seleccionados en función de los aspectos analizados.

\section{Resultados}

\section{Muestras de los estudios}

Tras analizar las muestras de los estudios, puede afirmarse que la etapa educativa en la que se ha realizado un mayor número de investigaciones es la Educación Superior, incluyendo a alumnado que cursa estudios de grado, máster, doctorado o formación profesional. En el $50 \%$ de los estudios analizados las muestras incluyen a alumnado de esta etapa educativa, aunque en algunos artículos las muestras estaban compuestas tanto por alumnado de Educación Superior como de Educación Secundaria. En el caso de Educación Secundaria y Educación Primaria, los datos obtenidos son bastante parejos. Sin embargo, existe un mayor el número de estudios con muestras compuestas por alumnado de Educación Primaria (18,42 \%) en comparación con los centrados en alumnado de Secundaria $(15,79 \%)$. Por último, la etapa educativa en la que menos investigaciones se han realizado sobre la influencia de las TIC en la instrucción en escritura es Educación Infantil, ya que solamente se encontró un estudio (McKenney y Voogt, 2009) en el que la muestra incluyera a alumnado de estas edades.

Asimismo, se analizaron las muestras con el fin de conocer cuántos estudios incluyen a alumnado con dificultades de aprendizaje. Tras dicho análisis, se observó que solo 6 de los 76 estudios incluían alumnado con dificultades de aprendizaje, tratándose en la mayor parte de las ocasiones de estudiantes con dislexia, como en los artículos de Beers et al. (2018) o Thompson et al. (2018). También se mostró que solo en tres estudios (Baker y Lastrapes, 2019; Palermo y Wilson, 2020; Woodrich y Fan, 2017) se incluye a alumnos en riesgo de deprivación social, haciendo referencia a estudiantes con un nivel socioeconómico bajo o de distinto origen étnico.

Otro dato llamativo derivado del análisis de las muestras es que en el 39,47 \% de los estudios la aplicación de las herramientas tecnológicas en la instrucción en escritura se realizaba para la enseñanza de una lengua extranjera. Sobre este aspecto, es preciso destacar que en la mayoría de estos estudios los alumnos eran estudiantes de inglés, aunque también se encontraron referencias al aprendizaje otros idiomas como el francés (García et al., 2019) o el árabe (Khan et al., 2019). Por último, cabe destacar que en 6 estudios no se especificaban las características de las muestras.

\section{Recursos tecnológicos empleados y variables evaluadas}

Para analizar los recursos tecnológicos empleados en los distintos artículos empíricos, en primer lugar, se realizó una separación de los mismos en dos tipologías. Por un lado, las herramientas tecnológicas diseñadas para la instrucción de procesos de orden superior de escritura. Y, por otro lado, herramientas tecnológicas diseñadas para apoyar aspectos mecánicos de la escritura (ortografía, gramática, vocabulario, etc.).

El primer resultado hallado fue que en el $65,79 \%$ de los estudios se empleaban herramientas tecnológicas para apoyar y/o mejorar aspectos mecánicos de la escritura, mientras que el número de herramientas destinadas a la enseñanza y/o mejora de procesos de orden superior y estrategias de escritura era mucho más reducido (34,21\%). Este aspecto coincide con los resultados hallados en la revisión empírica de Strobl et al. (2019), en la que se analizan los recursos tecnológicos existentes para apoyar la instrucción en escritura dentro de las etapas de Educación Secundaria y Educación Superior.

Tras este análisis general, se realizó un análisis más exhaustivo de cada tipología. Respecto a las herramientas digitales destinadas a la enseñanza de procesos de orden superior, se observó que el mayor número de herramientas empleadas eran Sistemas de Tutoría Inteligente (STI), en los cuales se aborda la instrucción de la escritura desde un enfoque procesual, dando importancia a la enseñanza de los procesos de planificación, textualización y revisión, tal y como aparece reflejado en la Tabla 1. Sin embargo, algunos de los STI encontrados se centraban en la enseñanza de habilidades específicas de escritura, destacando las relacionadas con la escritura de resúmenes, como en el artículo de Sung et al. (2016). Los resultados obtenidos en estos estudios mostraron mejoras significativas en la calidad textual de los estudiantes.

Dentro de esta tipología de herramientas tecnológicas, también se encontraron sistemas AWE, focalizados únicamente en la evaluación del proceso de revisión, y diversos softwares y plataformas digitales, focalizados especialmente en la enseñanza de 
Tabla 1. Tabla sintetizada de recursos tecnológicos para la enseñanza de aspectos de orden superior

\begin{tabular}{|c|c|c|c|}
\hline Autor y año & Instrumentos & $\begin{array}{c}\text { Tipo de } \\
\text { instrumento }\end{array}$ & Procesos \\
\hline $\begin{array}{l}\text { Cotos et al. (2020); Hilbert (2019); Li et al. } \\
\text { (2015); Palermo y Wilson (2020) }\end{array}$ & $\begin{array}{l}\text { - Research Writing Tutor (RWT) } \\
\text { - NC Write } \\
\text { - ProWritingAid } \\
\text { - Criterion }\end{array}$ & AWE & Revisión \\
\hline $\begin{array}{l}\text { Crossley et al. (2013); Holdich y Chung } \\
\text { (2003); Proske et al. (2012); Roscoe y McNa- } \\
\text { mara (2013); Rowley y Meyer (2003) }\end{array}$ & $\begin{array}{l}\text { - Writing Pal (W-Pal) } \\
\text { - Escribo } \\
\text { - Harry } \\
\text { - Computer Tutor for Writers (CTW) } \\
\end{array}$ & STI & $\begin{array}{l}\text { Planificación, } \\
\text { textualización } \\
\text { y revisión }\end{array}$ \\
\hline $\begin{array}{l}\text { Benetos y Bétrancourt (2020); Fronzke } \\
\text { (2005); Sung et al. (2016) }\end{array}$ & $\begin{array}{l}\text { - STI basado en el análisis semántico latente } \\
\text { - Summary Street } \\
\text { - C-SAW }\end{array}$ & STI & $\begin{array}{l}\text { Habilidades } \\
\text { específicas }\end{array}$ \\
\hline $\begin{array}{l}\text { Álvarez y Andueza (2017); Goldenberg et al. } \\
\text { (2011); González-Álvarez (2018); Liu et al. } \\
\text { (2012); Warren et al. (2008) }\end{array}$ & $\begin{array}{l}\text { - RedacText } 2.0 \\
\text { - LEA lab } \\
\text { - May's Writing Meadow } \\
\text { - Anytown } \\
\text { - Writing Matters } \\
\end{array}$ & $\begin{array}{l}\text { Plataforma } \\
\text { Software }\end{array}$ & $\begin{array}{l}\text { Planificación, } \\
\text { textualización } \\
\text { y revisión }\end{array}$ \\
\hline $\begin{array}{l}\text { Challob et al. (2016); Knight et al. (2020); } \\
\text { Ranalli et al. (2019); Yamac (2020) }\end{array}$ & $\begin{array}{l}\text { - Viber } \\
\text { - AcaWriter } \\
\text { - CyWrite } \\
\text { - SADIWE }\end{array}$ & Software & $\begin{array}{l}\text { Planificación } \\
\text { y revisión }\end{array}$ \\
\hline
\end{tabular}

Nota: Sistemas de Tutoría Inteligente (STI); Sistemas de Evaluación de Escritura Automatizada (AWE)

Tabla 2. Tabla sintetizada de recursos tecnológicos para la enseñanza de aspectos mecánicos

\begin{tabular}{|c|c|c|c|}
\hline Autor y año & Instrumentos & $\begin{array}{l}\text { Tipo de } \\
\text { instrumento }\end{array}$ & Variables \\
\hline $\begin{array}{l}\text { Bailey y Lee (2020); Cowan et al. (2014); Kim } \\
\text { (2018); Morgado et al. (2020); Murdoch y } \\
\text { Kang (2019); O'Rourke et al. (2020); Yeh et } \\
\text { al. (2014) }\end{array}$ & $\begin{array}{l}\text { - Grammarly } \\
\text { - Microsoft Office Word } \\
\text { - White Smoke } \\
\text { - Annotation Editor } \\
\text { - FCK Editor } \\
\text { - ESL Writing Tutor } \\
\text { - English Grammatical Error Detection } \\
\text { (GED) }\end{array}$ & $\begin{array}{l}\text { Software } \\
\text { Detector } \\
\text { de errores }\end{array}$ & Gramática \\
\hline $\begin{array}{l}\text { Bailey y Lee (2020); Kim (2018); Murdoch y } \\
\text { Kang (2019); O'Rourke et al. (2020) }\end{array}$ & $\begin{array}{l}\text { - Grammarly } \\
\text { - Microsoft Office Word } \\
\text { - White Smoke }\end{array}$ & $\begin{array}{l}\text { Software } \\
\text { Detector } \\
\text { de errores }\end{array}$ & $\begin{array}{l}\text { Gramática } \\
\text { y ortografía }\end{array}$ \\
\hline $\begin{array}{l}\text { Duman y Göcen (2015); Yamac y Ulusoy } \\
(2016)\end{array}$ & - Storytelling & Plataforma & $\begin{array}{c}\text { Vocabulario } \\
\text { y organización }\end{array}$ \\
\hline $\begin{array}{l}\text { Al-Jarf (2013); Bosseler y Massaro (2003); } \\
\text { Owen (2008); So (2019) }\end{array}$ & $\begin{array}{l}\text { - Actividades Fonf } \\
\text { - Baldi } \\
\text { - RCampus } \\
\text { - MNS Messenger }\end{array}$ & Plataformas & Vocabulario \\
\hline $\begin{array}{l}\text { Bailey y Lee (2020); Baker y Lastrapes (2019); } \\
\text { Fadhilawati et al. (2020); Luna et al. (2020); } \\
\text { Rahimi y Yadollahi (2017); Zou y Xie (2018) }\end{array}$ & $\begin{array}{l}\text { - Padlet } \\
\text { - Facebook } \\
\text { - Google Docs } \\
\text { - MyStoryTrees } \\
\text { - Story Jumper } \\
\end{array}$ & $\begin{array}{l}\text { Plataformas para } \\
\text { escritura } \\
\text { colaborativa }\end{array}$ & $\begin{array}{l}\text { Ortografía, } \\
\text { gramática, } \\
\text { vocabulario }\end{array}$ \\
\hline $\begin{array}{l}\text { Haziq et al. (2009); Tanimoto (2016); Thomp- } \\
\text { son et al. (2018) }\end{array}$ & $\begin{array}{l}\text { - Lecciones HAWK } \\
\text { - MyLexics }\end{array}$ & $\begin{array}{l}\text { Lecciones } \\
\text { en ordenador }\end{array}$ & $\begin{array}{l}\text { Letras, palabras, } \\
\text { sílabas y conciencia } \\
\text { fonológica }\end{array}$ \\
\hline
\end{tabular}

los procesos de planificación y revisión. En este caso, los resultados indican que con estos sistemas se dio un aumento en el pensamiento metacognitivo y en la motivación.

Por otro lado, respecto a las herramientas tecnológicas focalizadas en apoyar los aspectos mecánicos de la escritura, se halló un gran número de softwares destinados a la detección de errores gramaticales. Un aspecto llamativo fue el tipo de alumnado con el que se empleaban, ya que 5 de los 8 detectores de errores gramaticales reflejados en la Tabla 2 se utilizaron para la enseñanza de una lengua extranjera. Los estudios muestran que estos recursos mejoraron la calidad textual, reduciendo significativamente el número de errores.

En línea con los detectores de errores, los recursos digitales que más aparecieron recogidos en los distintos estudios revisados fueron las plataformas digitales, destacando entre ellas las destinadas a la escritura colaborativa, que tienen la ventaja de permitir al alumnado proporcionar retroalimentación a sus pares, como se ha mostrado en estudios como el de Zou y Xie (2018). En las 
plataformas digitales halladas, se observó que éstas se emplearon para evaluar principalmente las variables de ortografía, gramática y vocabulario, siendo esta última la más evaluada por los estudios. De este modo, teniendo en cuenta los datos obtenidos sobre las plataformas y los detectores de errores, la gramática es la variable más evaluada junto al vocabulario.

Por último, cabe destacar la existencia de herramientas que fomentan el desarrollo de la conciencia fonológica como las presentadas en los estudios de Thompson et al. (2018) y Haziq et al. (2009). Ambas herramientas contienen una serie de lecciones centradas en la enseñanza progresiva de letras (tanto mayúsculas como minúsculas), sílabas y palabras completas. Además, otro aspecto común entre estas dos herramientas ha sido su implementación en muestras que incluían estudiantes con dificultades de aprendizaje, destacando en dos de los estudios la presencia de alumnado con dislexia. Los resultados mostraron que el empleo de la tecnología influía de forma positiva sobre la conciencia fonológica, la ortografía, la morfología y la gramática.

\section{Discusión}

El objetivo del presente estudio fue realizar una revisión sistemática de artículos empíricos centrados en el empleo de herramientas digitales diseñadas para mejorar la calidad textual de las composiciones escritas del alumnado de las diferentes etapas educativas, diferenciándolas en dos tipologías: herramientas centradas en procesos de alto nivel cognitivo o en procesos de bajo nivel cognitivo. A continuación, se discuten los resultados hallados en torno a los distintos aspectos analizados.

Por un lado, en lo que respecta a las muestras participantes en los distintos artículos analizados, pueden destacarse tres aspectos. En primer lugar, los resultados ponen de manifiesto que la Educación Superior es la etapa educativa en la que se han realizado más estudios para comprobar la efectividad de las TIC en la instrucción en escritura. Este dato difiere de estudios previos centrados en investigar la instrucción en escritura de forma general, como la revisión realizada por Juzwik et al. (2006), en la que se muestra que la instrucción en escritura durante las últimas décadas se ha estudiado en mayor medida en la etapa de Educación Primaria. Sin embargo, teniendo en cuenta que en Educación Primaria el alumnado comienza a desarrollar en mayor medida la competencia escrita, sería de gran utilidad realizar más investigaciones que incluyan la utilización de las TIC con alumnos de estas edades, contribuyendo al buen proceso de adquisición de la escritura desde edades tempranas.

En segundo lugar, los resultados indican que el número de estudios cuyas muestras estaban compuestas por estudiantes con dificultades de aprendizaje o por alumnado en riesgo de deprivación social era muy reducido, aspecto que también se menciona en meta-análisis como el realizado por Little et al. (2018). Dado que los alumnos y alumnas que se encuentran en desventaja social suelen mostrar un menor rendimiento en escritura, que en ocasiones deriva en el abandono de los estudios (Bonal y Scandurra, 2019; Kim et al., 2015), este dato puede resultar de interés para sentar las bases de futuras investigaciones en las que se muestren cuáles son las ventajas que produce en este tipo de alumnado la introducción de las nuevas tecnologías en los programas de instrucción en escritura.

El último aspecto destacado en relación con las muestras de los estudios revisados es la gran utilización de las TIC para realizar la instrucción en escritura en una lengua extranjera. Pese a que en los estudios revisados se hace referencia a varios idiomas como el árabe o el francés, la mayor parte de este porcentaje se refiere al aprendizaje del inglés. Ello apoya los resultados hallados en investigaciones previas como la revisión empírica de Strobl et al. (2019), en la que un alto porcentaje de las herramientas tecnológicas localizadas se centraban en la enseñanza del inglés, atendiendo a aspectos mecánicos de la escritura como la gramática o la ortografía.

Por otro lado, respecto a los recursos tecnológicos empleados y las variables evaluadas, uno de los principales hallazgos se relaciona con el número de herramientas tecnológicas destinadas a la enseñanza de procesos de orden superior que han sido localizadas. A pesar de que la enseñanza de este tipo de procesos es beneficiosa para lograr el buen desarrollo de la competencia escrita (Koster et al., 2015), el número de herramientas tecnológicas destinadas a este fin es muy reducido en comparación con el número de herramientas centradas en aspectos mecánicos. Además, dentro de esta última tipología, la mayor parte de las herramientas son correctores de ortografía y gramática que, junto al vocabulario, son las variables más evaluadas en los estudios. A su vez, estos datos, apoyan los resultados hallados en revisiones previas como las realizadas por Limpo et al. (2020), Rodríguez et al. (2019) y Strobl et al. (2019) en las que se han analizado los efectos de las nuevas tecnologías en los programas de instrucción en escritura.

\section{Conclusiones}

Los estudios sobre desarrollo y aplicación de herramientas tecnológicas en el aprendizaje de la escritura son muy desiguales entre las diferentes etapas educativas. El número más significativo de herramientas tecnológicas de la instrucción en escritura se da en la etapa de Educación Superior siendo, por el contrario, casi inexistente en la Educación Infantil, cumpliendo con la primera hipótesis del estudio. En general, dichas herramientas se han aplicado en población normalizada, con un pequeño porcentaje dedicado a alumnado con dificultades de aprendizaje.

El número de estudios sobre herramientas tecnológicas destinadas a la enseñanza de estrategias o procesos de orden superior es muy reducido comparado con las herramientas diseñadas para apoyar los aspectos mecánicos de la escritura, tal y como se había planteado en la segunda hipótesis del estudio. Esta revisión muestra que la mayoría de las herramientas tecnológicas focalizadas en aspectos mecánicos de escritura son detectores de errores gramaticales. Asimismo, entre aquellas desarrolladas para instruir en procesos cognitivos de orden superior, la mayor parte son STI centrados en la enseñanza de los procesos de planificación, textualización y revisión.

La presentación y el análisis de distintos recursos tecnológicos aplicados a la instrucción en escritura resulta de utilidad a nivel educativo tanto para docentes en formación como en ejercicio. El conocimiento de las características concretas de distintas herramientas digitales puede favorecer su incorporación en el aula, ya que al conocer las variables que pueden ser evaluadas con cada herramienta tecnológica, los docentes podrán seleccionar la más adecuada para solventar los problemas o necesidades concretas que sean detectadas en el aula. Además, las TIC pueden facilitar la labor docente evitando dificultades propias del aula como, por ejemplo, la falta de tiempo para atender de manera individualizada al alumnado. Algunas herramientas digitales, también ofrecen la posibilidad de proporcionar retroalimentación entre pares, favoreciendo la motivación hacia la tarea y el refuerzo del aprendizaje.

Finalmente, en base a los resultados obtenidos, cabe el plantearse como futuras líneas de investigación, el análisis más exhaustivo de los recursos TIC disponibles para la enseñanza de la escritura, tanto a nivel mecánico como sustantivo, en torno a sus microcomponentes a nivel de aprendizaje del alumnado, y de secuenciación y elaboración instruccional. Esto, vinculado a los efectos que su uso provoca a nivel de rendimiento de escritura del alumnado y del desarrollo de posibles variables moduladoras de la escritura, como las actitudes hacia la escritura, su autoeficacia, etc., ofrecería datos de indudable valor en la explicación de la efectividad de las TIC para la enseñanza de la escritura. 


\section{Agradecimientos}

Esta investigación ha sido financiada mediante el proyecto EDU2015-67484-P (MINECO/FEDER), concedido a la Dra. Raquel Fidalgo.

Contribution individual de las autoras

MVGL ha sido responsable de realizar el trabajo de campo, el análisis y la redacción. RFR, ha realizado el diseño del estudio y del análisis y la revisión del manuscrito.

\section{Referencias}

Al-Jarf, R. (2013). Enhancing freshman students' performance with online reading and writing activities. Proceedings of eLearning and Software for Education, 2, 524-530. https://doi.or$\mathrm{g} / 10.12753 / 2066-026 \mathrm{X}-13-193$

Álvarez, T., \& Andueza, A. (2017). Uso de tecnologías para facilitar el proceso de composición escrita: análisis defecto de la plataforma RedacText 2.0 en la calidad en los textos académicos escritos por estudiantes de Magisterio. Revista Complutense de Educación, 28(1), 283-305. https://doi.org/10.5209/rev_ RCED.2017.v28.n1.49449

Andrade, M. \& Moreno, D. (2017). Leer y escribir en tiempos de las nuevas tecnologías de la información y la comunicación. Revista de Tecnología de la Información y de la Comunicación en la Educación, 11(1), 55-66. http://dx.doi.org/10.5209/rev_ RCED.2017.v28.n1.49449

Bailey, D., \& Lee, A. (2020). An Exploratory Study of Grammarly in the Language Learning Context: An Analysis of Test-Based, Textbook-Based and Facebook Corpora. TESOL, 15(2), 4-27.

Baker, S. \& Lastrapes, R. (2019). The writing performance of elementary students using a digital application. Interactive Technology and Smart Education, 16(4), 343-362. https://doi. org/10.1108/ITSE-08-2018-0057

Benetos, K., \& Bétrancourt, M. (2020). Digital Authoring Support for Argumentative Writing: What does it change? Journal of Writing Research, 12(1), 263-290. https://doi.org/10.17239/jowr2020.12.01.09

Beers, S. F., Berninger, V., Mickail, T., \& Abbott, R. (2018). Online Writing Processes in Translating Cognition into Language and Transcribing Written Language by Stylus and Keyboard in Upper Elementary and Middle School Students with Persisting Dysgraphia or Dyslexia. Learn Disabil, 23(2), 1-27. https:// doi.org/10.18666/LDMJ-2018-V23-I2-9008

Bolaño-García, M. (2017). Funciones de las herramientas multimedia interactivas para la enseñanza en educación preescolar. Praxis, 13(1), 17-24. https://dx.doi.org/10.21676/23897856.2063

Bonal, X., \& Scandurra, R. (2019). Equidad y Educación en España. Diagnóstico y prioridades. Globalisation, Education \& Social Policies.

Bosseler, A., \& Massaro, D. W. (2003). Development and Evaluation of a Computer-Animated Tutor for Vocabulary and Language Learning in Children with Autism. Journal of Autism and Developmental Disorders, 33(6), 653-672. https://doi.org/10.1023/ B:JADD.0000006002.82367.4f

Challob, A., Bakar, N., \& Latif, H. (2016). Collaborative Blended Learning Writing Environment: Effects on EFL students' writing apprehension and writing performance. English Language Teaching, 9(6), 229-241. http://dx.doi.org/10.5539/elt.v9n6p229

Cotos, E., Huffman, S., \& Link, S. (2020). Understanding graduate writers' interaction with and impact of the Research Writing Tutor during revision. Journal of Writing Research, 12(1), 187232. https://doi.org/10.17239/jowr-2020.12.01.07

Crossley, S. A., Vaner, L. K., Roscoe, R. D., \& McNamara, D. S. (2013). Using Automated índices of cohesión to evaluate an intelligent tutoring system and an automated writing evaluation system. In H. Chad, K. Yacef, J. Mostow, \& P. Pavlik (Eds.), Artificial Intelligence in Education (pp. 269-278). Springer
Duman, B., \& Göcen, G. (2015). The effect of the digital storytelling method pre-service teachers' creative writing skills. Anthropologist, 20(1), 215-222. https://doi.org/10.12973/eujer.10.1.13

Fadhilawati, D., Laksmita, D., \& Mansur, M. (2020). Using Padlet to increase the students' procedure text writing achievement. Exposure Journal, 9(2), 158-172. https://doi.org/10.26618/EXPOSURE.V912.3970

Fronzke, M., Kintsch, E., Caccamise, D., Johnson, N., \& Dooley, S. (2005). Summary Street ${ }^{\circledR}:$ Computer support for comprehension and writing. Journal of Educational Computing Research, 33(1), 53-80. https://doi.org/10.2190/DH8F-QJWM-J457-FQVB

Galvin, S., \& Greenhow, C. (2019). Writing on Social Media: a Review of Research in the High School Classroom. TechTrends, 64, 57-69. https://doi.org/10.1007/s11528-019-00428-9

García, G., Botero, M. A., Zhu, C., \& Questier, F. (2019). Complementing in-class language learning with voluntary out-ofclass MALL. Does training in self-regulation and scaffolding make a difference? Computer Assisted Language Learning, 32(1), 1-16. https://doi.org/10.1080/09588221.2019.1650780

Gillespie, A., \& Graham, S. (2014). A meta-análysis of writing interventions for students with learning disabilities. Exceptional Children, 80(4), 454-473. https://doi. org/10.1177/0014402914527238

Goldenberg, L., Meade, T., Midouhas, E., \& Cooperman, N. (2011). Impact of a technology-infused middle school writing program on sixth-grade students' writing ability and engagement. Middle Grades Research Journal, 6(2), 75-96.

González-Álvarez, P. (2018). Diseño de una plataforma virtual de autoaprendizaje de la escritura académica: fundamentación teórica y decisiones pedagógicas en la Universidad de Chile. Álabe, 17, 1-17. https://doi.org/10.15645/Alabe2018.17.7

Graham, S., Collins, A., \& Rigby-Wills, H. (2017). Writing Characteristics of Students With Learning Disabilities and Typically Achieving Peers: A Meta-Analysis. Exceptional Children, 83(2), 199-218. https://doi.org/10.1177/0014402916664070

Graham, S., Harris, K. R., \& McKeown, D. (2013). The writing of students with LD and meta-analysis of SRSD writing intervention studies: Redux. In L. Swanson, K. R. Harris, \& S. Graham (Eds.), Handbook of learning disabilities (pp. 405-438). New York.

Graham, S. \& Perin, D. (2007). A meta-analysis of writing instruction for adolescent students. Journal of Educational Psychology, 99, 445-476. https://doi.org/10.1037/0022-0663.99.3.445

Haziq, M., Hisham, S., \& Parumo, S. (2009). MyLexics: An Assistive Courseware for Dyslexic Children to Learn Basic Malay Language. Sigacces Newsletter, 95, 1-7. https://doi.org/10.1145/ 1651259.1651259.1651260

Hilbert, A. I. (2019). Systematic Literature Review of Automated Writing Evaluation as a Formative Learning Tool. In M. Scheffel, J. Broisin, V. Pammer-Schindler, A. Ioannou, \& J. Schneider (Eds.), Transforming Learning with Meaningful Technologies, (pp.199-212). Springer. https://doi.org/10.1007/978-3-03029736-7_15

Holdich, C. E., \& Chung, P. W. (2003). A computer tutor to assist children develop their narrative writing skills: conferencing with HARRY. International Journal of Human-Computer Studies, 59(5), 631-669. https://doi.org/10.1016/S1071-5819(03)00086-7

Juzwik, M. M., Curic, S., Wolbers, K., Moxley, K. D., Dimling, L., \& Shankland, R. K. (2006). Writing into the $21^{\text {st }}$ Century. An Overview of Research on Writing, 1999 to 2004. Written Communication, 23(4), 451-476. https://doi. org/10.1177/0741088306291619

Khan, D., Rehman, I., Ullah, S., Ahmad, W., Cheng, Z., Jabeen, G., \& Kato, H. (2019). A low-cost interactive writing board for primary education using distinct augmented reality markers. Sustainability, 11, 1-14. https://doi.org/10.3390/su11205720

Kim, V. (2018). Technology-Enhanced Feedback on Student Writing in the English-Medium Instruction Clssroom. 
English Teaching, 73(4), 29-53. https://doi.org/10.15858/engtea.73.4.201812.29

Kim, Y. S., Puranik, C., \& Otaiba, S. (2015). Developmental trajectories of writing skills in first grade. Examining the Effects of SES and Language and/or Speech Impairments. The Elementary School Journal, 115(4), 593-613. https://doi.org/00135984/2015/11504-0008

Knight, S., Shibani, A., Abel, S., Gibson, A., Ryan, P., Sutton, N., Wight, R., Lucas, C., Sándor, A., Kitto, K., Liu, M., Vijay, R., \& Buckinham, S. (2020). AcaWriter a learning analytics tool for formative feedback on academic writing. Journal of Writing Research, 12(1), 141-186. https://doi.org/10.17239/jowr2020.12.01.06

Koster, M., Tribushinina, E., De Jong, P., \& Van den Bergh, H. (2015). Teaching Children to Write: A Meta-analysis of Writing Intervention Research. Journal of Writing Research, 7(2), 249-274. https://doi.org/10.17239/jowr-2015.07.02.02

Kwak, S. (2017). Approaches Reflected in Academic Writing MOOCs. International Review of Research in Open and Distributed Learning, 18(3), 138-155. https://doi.org/10.19173/irrodl. v18i3.2845

Leu, D. J., Kinzer, C. K., Coiro, J., Castek, J., \& Henry, L. A. (2013). New literacies: a dual-level theory of the changing nature of literacy. In D. E. Alvermann, N. J. Unrau, \& R. B. Ruddell (Eds.), Theoretical Models and Processes of Reading (pp. 1150-1180). International Reading Association.

Li, J., Link, S., \& Hegelheimer, V. (2015). Rethinking the role of automated writing evaluation (AWE) feedback in ESL writing instruction. Journal of Second Language Writing, 27, 1-18. https:// doi.org/10.1016/j.jslw.2014.10.004

Limpo, L., Nunes, A., \& Coelho, A. (2020). Introduction to the special issue on 'Technology-based writing instruction: A collection of effective tools'. Journal of Writing Research, 12(1), 1-7. https://doi.org/10.17239/jowr-2020.12.01.01

Little, C., Clark, J., Tani, N., \& McDonald, C. (2018). Improving writing skills through tecnology-based instruction: A meta-analysis. Review of Education, 6(2), 183-201. https://doi. org/10.1002/rev3.3114

Liu, Y. C., Lee, W. C., Huang, T. H, \& Hsieh, H. M. (2012). Improving students' Chinese writing abilities in Taiwan with the “conditioned writing system". TOJET, 11(3), 189-201.

Luna, M., Villalón, R., Mateos, M., \& Martín, E. (2020). Improving University Argumentative Writing through Online Training. Journal of Writing Research, 12, 233-262. https://doi. org/10.17239/jowr-2020.12.01.08

McKenney, S., \& Voogt, J. (2009). Designing technology for emergent literacy: the PictoPal initiative. Computers $\mathcal{E}$ Education, $52,719-729$.

Ministerio de Educación y Formación Profesional (2019). TALIS 2018. Estudio internacional de la enseñanza y del aprendizaje. Informe español. Secretaría General Técnica.

Murdoch, Y. D., \& Kang, A. (2019). Direct instruction and use of online English writing software on EMI Class-takers' self-efficacy. Instrumental Journal of Contents, 15(4), 97-106. https://doi. org/10.5392/IJoC.2019.15.4.097

O'Rourke, L., Conelly, V., Barnett, A., \& Alfonso, O. (2020). Use os spellcheck in text production by college students with dyslexia. Journal of Writing Research, 12(1), 35-62. https://doi. org/10.17239/jowr-2020.12.01.03

Owen, H. (2008). Learners re-shaping learning landscapes: New directions for old challenges? Proceedings ascilite Melbourne 2008, 715-723. ASCILITE.

Palermo, C., \& Wilson, J. (2020). Implementing Automated Writing Evaluation in Different Instructional Contexts: A Mixed-Methods Study. Journal of Writing Research, 12(1), 63108. https://doi.org/10.17239/jowr-2020.12.01.04
Perin, D., \& Holschuh, J. P. (2019). Teaching Academically Underprepared Postsecondary Students. Review of Research in Education, 43, 363-393. https://doi.org/10.3102/0091732X18821114

Proske, A., Narciss, S., \& McNamara, D. S. (2012). Computer-based scaffolding to facilitate students' development of expertise in academic writing. Journal of Research in Reading, 35(2), 136152. https://doi.org/10.1111/j.1467-9817.2010.01450.x

Rahimi, M., \& Yadollahi, S. (2017). Effects of offline vs. online digital storytelling on the development of EFL learners' literacy skills. Cogent Education, 4, 1-13. https://doi.org/10.10.1080/233 1186X.2017.1285531

Ranalli, J., Feng, H., \& Chukharev-Hudilainen, E. (2019). The affordances of process-tracing technologies for supporting L2 writing instruction. Language Teaching and Technology Forum, 23(2), 1-11. https://doi.org/10125/44678

Rodríguez, L., Rodríguez, C. \& Fidalgo, R. (2019). Nuevos entornos de aprendizaje para la escritura: Sistemas de Tutoría Inteligente. Papeles del Psicólogo, 40(2), 133-140. https://doi. org/10.23923/pap.psicol2019.2895

Roscoe, R. D., \& McNamara, D. S. (2013). Writing Pal: Feasibility of an intelligent writing strategy tutor in the high school classroom. Journal of Educational Psychology, 105(4), 1010-1025. https://doi.org/10.1037/a0032340

Rowland, A., Smith, S. J., \& Lowrey, A. (2020). Pairing Technology with 6 traits of writing instruction. Journal of Special Education Technology, 20(10), 1-8. https://doi. org $/ 10.1177 / 0162643420945600$

Rowley, K., \& Meyer, N. (2003). The effect of a computer tutor for writers on student writing achievement. Journal of Educational Computing Research, 29(2), 169-187. https://doi.org/10.2190/3WVD-BKEY-PK0D-TTR7

So, L. (2019). A case Study on focus on form with web content in L2 writing instruction at university students' level. Studies in Foreign Language Education, 33(1), 25-53. http://dx.doi. org/10.16933/sfle.2019.33.1.25

Suárez-Palacio, P. A., Vélez-Múnera, M., \& Londoño-Vásquez, D. A. (2018). Las herramientas y recursos digitales para mejorar los niveles de literacidad y el rendimiento académico de los estudiantes de primaria. Revista Virtual Universidad Católica del Norte, 54, 184-198.

Sung, Y. T., Liao, C. N., Chang, T. H., Chen, C. L., \& Chang, K. E. (2016). The effect of online summary assessment and feedback system on the summary writing on 6th graders: The LSA-based technique. Computers $\mathcal{E}$ Education, 95, 1-18. https://doi.org/10.1016/j.compedu.2015.12.003

Strobl, C., Ailhaud, E., Benetos, K., Devitt, A., Kruse, O., Proske, A., \& Rapp, C. (2019). Digital support for academic writing: A review of technologies and pedagogies. Computers $\mathcal{E}$ Education, 131, 33-48. https://doi.org/10.1016/j.compedu.2018.12.005

Tanimoto, S., Thompson, R., Berninger, V. W., Nagy, W., \& Abbott, R. D. (2016). Computerized Writing and Reading Instruction for Students in Grades 4 to 9 With Specific Learning Disabilities Affecting Written Language. Journal of Computer Assisted Learning, 31(6), 671-689. https://doi.org/10.1111/ jcal.12110

Thompson, R., Tanimoto, S., Dawn, R., Geselowitz, K., Kawena, K., Nielsen, K., Nagy, W., Abbott, R., Raskind, M., \& Berninger, V. (2018). Effective Instruction for Persisting Dyslexia in Upper Grades: Adding Hope Stories and Computer Coding to Explicit Literacy Instruction. Education and Information Technologies, 23(3), 1043-4068. https://doi.org/10.1007/s10639-0179647-5

Venegas-Ramos, L., Luzardo, H. J., \& Pereira, A. (2020). Conocimiento, formación y uso de herramientas TIC aplicadas a la Educación Superior por el profesorado de la Universidad Miguel de Cervantes. EDUTEC. Revista Electrónica de Tecnología Educativa, 71, 35-52. https://doi.org/10.21556/edutec.2020.71.1405 
Wang, S. K., Hsu, H. Y., Campbell, T., Coster, D. C., \& Longhurst, M. (2014). An investigation of middle school science teachers and students use of technology inside and outside of classrooms: considering whether digital natives are more technology savvy than their teachers. Educational Technology Research and Development, 62(6), 637-662.

Warren, S. J., Dondlinger, M. J., \& Barab, S. A. (2008). A MUVE Towards PBL Writing: effects of a digital learning environment designed to improve elementary student writing. JRTE, 4(1), 113-140.

Woodrich, M., \& Fan, Y. (2017). Google Docs as a tool for collaborative writing in the middle school classroom. Journal of Information Technology Education: Research, 16, 391-410. https:// doi.org/10.28945/3870
Yamac, A., Öztürk, E., \& Mutlu, N. (2020). Effect of digital writing instruction with tablets on primary school students' writing performance and writing knowledge. Computers $\mathcal{E}$ Education, 157, 1-19. https://doi.org/10.1016/j.compedu.2020.103981

Yamac, A., \& Ulusoy, M. (2016). The effect of digital storytelling in improving the third graders' writing skills. International Electronic Journal of Elementary Education, 9(1), 59-86.

Zou, D., \& Xie, H. (2018). Flipping an English writing class with technology-enhanced just-in-time teaching and peer instruction. Interactive Learning Environments, 27(8), 1127-1142. https:// doi.org/10.1080/10494820.2018.1495654 